\title{
Value Added Impact of Both Point-of-Care and Laboratory Lactic Acid Analysis When Emergently Evaluating Cancer Patients
}

\author{
Georges T. Bouobda - Carmen E. Gonzalez - Ron A. Phipps • \\ Lavinia P. Middleton D
}

Received: April 7, 2020 / Published online: June 2, 2020

(C) The Author(s) 2020

\begin{abstract}
Introduction: Cancer patients are immunosuppressed and may present to an emergency department with atypical symptoms. In the emergency setting, it is important ascertain rapidly if lactic acid levels are high, either due to sepsis or tumor lysis syndrome, to effectively manage symptoms. Therefore, it is critical to determine the blood lactic acid level to timely identify who is at risk of sepsis and provide early intervention. We have compared blood lactic acid concentrations (BLAC) in cancer patients obtained by point-of-care testing (POCT) and those measured by laboratory analysis in blood samples drawn within a short time of each other.
\end{abstract}

Methods: This was a retrospective study in cancer patients whose BLAC had been

Digital Features To view digital features for this article go to https://doi.org/10.6084/m9.figshare.12348110.

Electronic Supplementary Material The online version of this article (https://doi.org/10.1007/s40487020-00118-0) contains supplementary material, which is available to authorized users.

G. T. Bouobda

Morehouse School of Medicine, Atlanta, GA, USA

C. E. Gonzalez · R. A. Phipps · L. P. Middleton $(\bowtie)$ University of Texas MD Anderson Cancer Center, Houston, TX, USA

e-mail: lpmiddleton@mdanderson.org determined by POCT and laboratory analysis. Only those patients who had blood withdrawn for both testing methods within a 2-h timeframe were included in the study. Regressions were performed together with an analysis categorizing the BLAC from both testing methods.

Results: A total of 274 patients met the criteria for the study. The BLAC from POCT correlated well with the values from laboratory testing $(R=0.925)$. Categorization of BLAC showed that $88.32 \%$ of the patients had BLAC that directly matched between the two tests; 28 (10.22\%) patients had a normal BLAC according to laboratory analysis but a high BLAC on POCT; and four (1.46\%) patients had a high BLAC according laboratory analysis but normal BLAC on POCT.

Conclusions: There was a high correlation between POCT and laboratory analysis values of BLAC in cancer patients, with the results from both testing methods agreeing $96 \%$ of the time. This finding suggests that POCT would suffice in most cases. Importantly, in $2 \%$ of the cancer patients who presented emergently, BLAC determined by POCT and laboratory analysis did not agree. Therefore, in subsequent decision-making, we recommend that if sepsis is suspected and BLAC determined by POCT is normal, nucleic acids, proteins, circulating cells, and interleukin-3 levels should also be obtained by POCT to confirm sepsis and/or rule out tumor lysis syndrome in patients with cancer. 
Keywords: Cancer; Emergency department; Lactic acid; Point-of-care; Sepsis

\section{Key Summary Points}

Immunocompromised patients with cancer may present to an emergency department with atypical presentations.

In the emergency setting, it is important ascertain rapidly if lactic acid levels are high, either due to sepsis or tumor lysis syndrome.

If sepsis is suspected and point-of-care testing (POCT) for lactic acid is normal, POC test results for nucleic acid, proteins, circulating cells, and interleukin-3 levels should also be obtained to confirm sepsis and/or rule out tumor lysis syndrome in this vulnerable population.

\section{INTRODUCTION}

Lactate is a metabolite resulting from the fermentation of glucose when tissues lack or cannot use oxygen for the oxidative phosphorylation of glucose into energy. High levels of lactate in the blood, either from tumor lysis syndrome or sepsis, can precipitously cause death [1]. This association is even more important in cancer patients because not only do cancerous cells secrete lactate even in an abundance of oxygen [2], but cancer patients may also be immunosuppressed due to the cancer and its treatment, rendering them more susceptible to systemic infection. Blood lactate concentration has been shown to reflect the progression of disease [3] and is thus a very important factor to consider when making a diagnosis in critically ill patients, especially those with cancer.

Laboratory analytical techniques, blood gas analyzers, point-of-care (POC) devices, and other methods are used to measure the lactate level in the blood. The recent breakthrough in the design of electrochemical biosensors, fieldeffect transistors, and surface plasmon resonance sensors has led to massive improvements in the development of POC devices. The processing technology used by new POC devices allow for a cost-effective, rapid, and efficient measurement of not only lactic acid levels but also of nucleic acid, proteins, and cells [4-7].

Previous studies have shown that POC devices are able provide reliable measurements of lactate levels in delivery rooms and emergency departments (ED) [8-10]. In the study reported here, we shift the focus to cancer patients who not only have cells producing more lactate but may be receiving treatment making them more susceptible to systemic infections.

The objective of this study was to compare blood lactic acid concentrations (BLAC) in cancer patients determined from point-of-care testing (POCT) using the i-STAT (Abbot Point of Care Inc., Princeton, NJ, USA) POC device with BLAC determined via laboratory analysis of concurrently acquired blood lactic acid samples. The Abbot POC device was used solely because it is the device that has been used for blood lactic acid measurements at the cancer hospital where the study was conducted. We determined the correlation between the two testing methods results to assess whether both test results are needed to triage patients with cancer. We hypothesize that POCT alone would provide an accurate measure of BLAC in cancer patients and due to the faster availability of the results, we anticipated better outcomes for cancer patients at risk of or suffering from sepsis as it has been shown that the timing of the intervention is a crucial factor in improving the outcomes of patients with sepsis [11]

\section{METHODS}

\section{Study Design and Selection of Participants}

This was a retrospective study with a sample of 2094 cancer patients from various clinical departments of a comprehensive cancer hospital. All of the patients presented to an ED of a comprehensive cancer center with acute symptoms within an 8-week period in 2018. At one point or another during this 8-week period, all patients underwent blood lactic acid testing, either by POCT with the i-STAT device, by 
laboratory analysis of samples, or both. Only those patients whose blood lactic acid levels had been tested by both POCT and concurrent laboratory analysis, with the blood drawing for the different tests carried out within $2 \mathrm{~h}$ of each other, were selected for inclusion in the statistical analysis.

This study is a laboratory-based quality improvement study based on anonymous patient data, and as such, not on research on human subjects, and is therefore exempt from review by our institution IRB.

\section{Data Collecting and Processing}

A chart review was conducted, and blood lactic acid test results and other useful demographics for the patients of interest were collected. Of the 2094 cancer patients who presented with acute symptoms within the 8-week study period, 274 (13.1\%) qualified for the analysis. Of these 274 patients, 13 had blood drawn for both POCT and laboratory analysis on two or more separate occasions within the 2-h timeframe. For these 13 patients, only the blood lactic acid values with the lowest time difference between the actual blood drawing for both testing methods were included in the analysis. One of the patients was removed from the regression analysis because the laboratory blood lactic acid test result had been quantified as a range rather than as a numerical value.

\section{Data Analysis}

A regression analysis using SPSS software release 23 (IBM Corp., Armonk, NY, USA) was performed between the blood lactic acid values obtained by POCT and those obtained by laboratory analysis. The Wilcoxon signed rank test was also used to compare POCT and laboratory lactic acid levels (Minitab version 18.1; Minitab, LLC, State College, PA, USA). For more details on the analysis, see the information file in the Electronic Supplementary Material. The data sample was further broken down into patients who had blood drawn for both the i-STAT POC device and laboratory analysis within $1 \mathrm{~h}, 30 \mathrm{~min}$, and $5 \mathrm{~min}$ of each other. Regression analysis was also performed on each of the three samples. Statistical analysis was performed to determine if for each patient the lactic acid concentrations obtained through both POCT and laboratory analysis fell into the same category since in our hospital the results from both testing methods are interpreted over different ranges. For each testing method, a BLAC could possibly fall into three categories: low, normal, and high. In our institution, for POCT, a BLAC of $\leq 0.9$ is considered low; a BLAC of $>0.9$ and $<1.7$ is noninclusive and considered to be normal; and a BLAC of $\geq 1.7$ is considered to be high. Similarly, for the laboratory testing, a BLAC of $\leq 0.5$ is considered to be low; a BLAC of $>0.5$ and $<2.2$ is non-inclusive and considered to be normal; and a BLAC $\geq 2.2$ is considered to be high.

\section{RESULTS}

\section{Characteristics of Study Subjects}

After chart reviews, 274 patients qualified for inclusion in the study. The age of this study population ranged from 6 to 87 years (mean $58.32,95 \%$ confidence interval [CI] 56.44-60.20 years). The sample comprised 146 (53.3\%) males and 128 females (46.7\%). All patients had a diagnosis of cancer (Table 1). The BLAC determined by POCT ranged from 0.4 to $14.0 \mathrm{mmol} / \mathrm{L}$ (mean $2.016,95 \%$ CI $1.788-2.244$ $\mathrm{mmol} / \mathrm{L})$; that determined by laboratory analysis ranged from 0.5 to $14.5 \mathrm{mmol} / \mathrm{L}$ (mean $2.244,95 \%$ CI $2.002-2.485 \mathrm{mmol} / \mathrm{L}$ ).

\section{Outcomes}

Regression analysis showed a strong correlation between blood lactic acid concentrations from POCT and those from laboratory testing ( $n=273$ patients, $R=0.925$ ) (Fig. 1 ). The trend in the blood lactic concentrations was similar whether the blood samples for both tests were drawn within $1 \mathrm{~h}$ of each other $(n=248$ patients, $R=0.970$ ) (Fig. 2), $30 \mathrm{~min}$ of each other ( $n=199$ patients, $R=0.982$ ) (Fig. 3), or 5 min of each other ( $n=57$ patients, $R=0.976$ ) (Fig. 4). 
Table 1 Demographics of the study population

\begin{tabular}{|c|c|}
\hline $\begin{array}{l}\text { Demographics of study } \\
\text { population }\end{array}$ & $\begin{array}{l}\text { Values (all subjects, } \\
n=274 \text { ) }\end{array}$ \\
\hline Age, years $($ mean $\pm S D)$ & $58.32 \pm 15.82$ \\
\hline Female, $n(\%)$ & $128(46.7)$ \\
\hline \multicolumn{2}{|c|}{ Most common cancer types, $n(\%)$} \\
\hline Leukemia & $54(19.7)$ \\
\hline Lymphoma & $25(9.1)$ \\
\hline Lung cancer & $25(9.1)$ \\
\hline Breast cancer & $24(8.8)$ \\
\hline Gastrointestinal cancer & $20(7.3)$ \\
\hline Sarcoma & $17(6.2)$ \\
\hline Multiple myeloma & $13(4.7)$ \\
\hline Prostate cancer & $11(4)$ \\
\hline Skin cancer & $10(3.6)$ \\
\hline Pancreatic cancer & $10(3.6)$ \\
\hline Bladder cancer & $9(3.3)$ \\
\hline Rectal cancer & $8(2.9)$ \\
\hline Myeloproliferative neoplasm & $7(2.6)$ \\
\hline Kidney cancer & $7(2.6)$ \\
\hline Ovarian cancer & $6(2.2)$ \\
\hline Esophageal cancer & $5(1.8)$ \\
\hline Uterine cancer & $4(1.5)$ \\
\hline
\end{tabular}

$S D$ Standard deviation

Analysis of the categorical range of the results revealed that two $(0.73 \%)$ patients were identified with low BLAC from both the laboratory analysis and POCT; 95 (34.67\%) patients were identified with normal BLAC from both testing methods; 81 (29.56\%) patients were identified with high BLAC from both testing methods; $64(23.36 \%)$ patients were identified with normal BLAC from the laboratory analysis and low BLAC from POCT; 28 (10.22\%) patients were identified with normal BLAC from the laboratory analysis and high BLAC from POCT; and four $(1.46 \%)$ patients were identified with high BLAC from the laboratory analysis and normal BLAC from POCT.

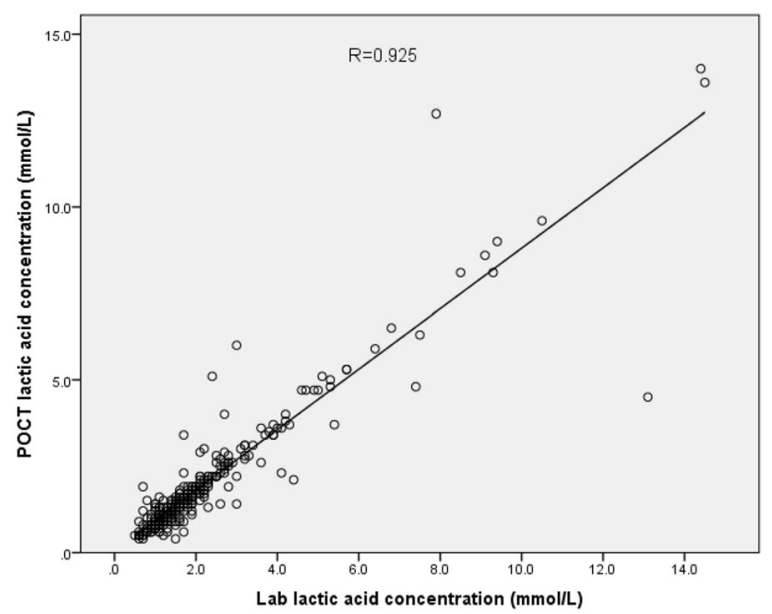

Fig. 1 Regression plot of blood lactic acid concentrations (BLAC) determined by point-of-care testing (POCT) and by laboratory analysis when the time interval between drawing blood samples for the two methods was $<2 \mathrm{~h}$

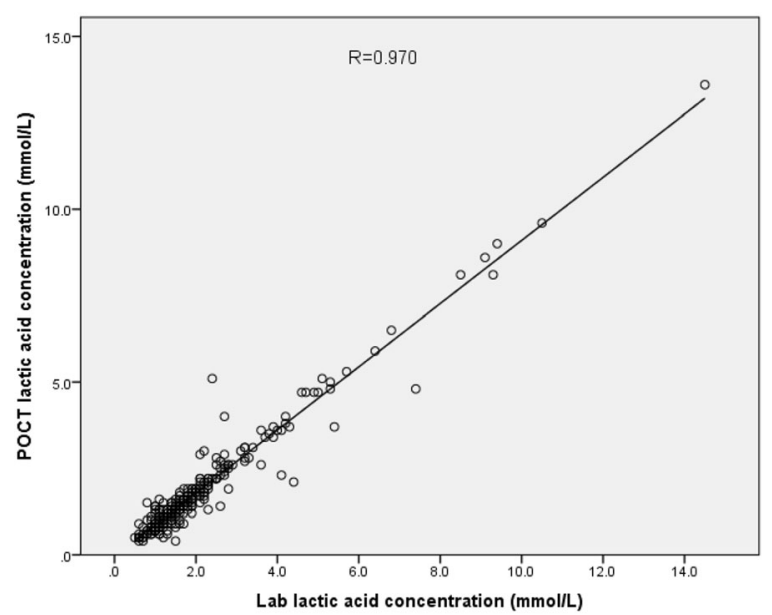

Fig. 2 Regression plot of BLAC determined by POCT and by laboratory analysis when the time interval between drawing blood samples for the two methods was $<1 \mathrm{~h}$

The median values for BLAC obtained from POCT and the laboratory analysis were 1.5 [Quartile $(Q) 1=1, Q 3=2.4$ ) and $1.4 \mathrm{mmol} / \mathrm{L}$ $(Q 1=1, Q 3=2.2)$, respectively.

\section{DISCUSSION}

In this study, there was a high correlation in BLAC in cancer patients determined by POCT and by laboratory analysis. However, it must be 


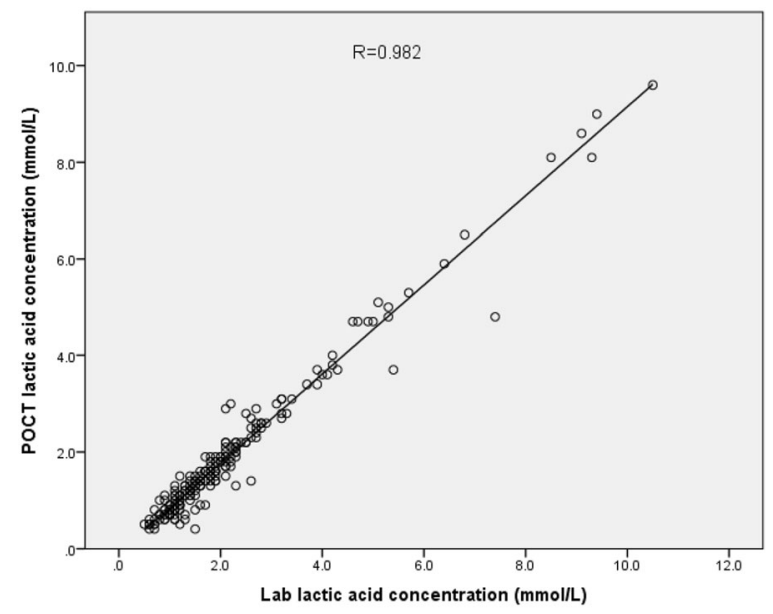

Fig. 3 Regression plot of BLAC determined by POCT and by laboratory analysis when the time interval between drawing blood samples for the two methods was $<30 \mathrm{~min}$

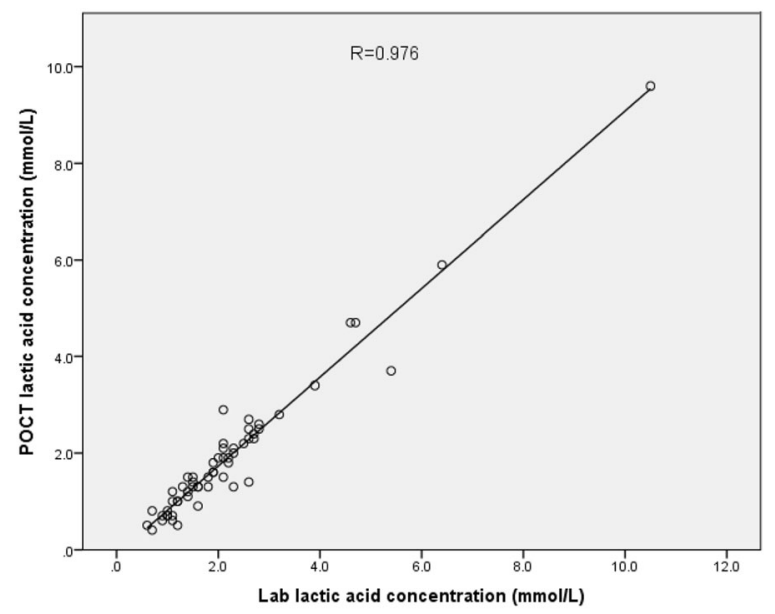

Fig. 4 Regression plot of BLAC determined by POCT and by laboratory analysis when the time interval between drawing blood samples for the two methods was $<5 \mathrm{~min}$

noted that in $2 \%$ of the cancer patients presenting emergently, there was disagreement between BLAC determined by POCT and laboratory analysis. Therefore, we recommend that if sepsis is suspected and BLAC determined by POCT is normal, tumor lysis syndrome should be considered. While in most cases there is no need to perform both POCT and laboratory analysis to obtain BLAC when triaging cancer patients, thus ensuring a timelier triage of the patient, in a minority of cases both tests are necessary. Our regression analysis in 273 cancer patients who had blood drawn for POCT and for concurrent laboratory analysis of BLAC within 2 $\mathrm{h}$ of each other showed a strong correlation between BLAC from both testing methods $(R=0.925)$. Taking into account that once blood is drawn, the BLAC will keep increasing due to red blood cell metabolism, we also performed regression analysis on BLAC determined by both POCT and laboratory analysis in blood samples drawn within $1 \mathrm{~h}$ of each other $(R=0.970), 30 \mathrm{~min}$ of each other $(R=0.982)$, and $5 \mathrm{~min}$ of each other $(R=0.976)$. The results of these regressions, similar to that for the $2-\mathrm{h}$ interval between the different analyses, showed a strong correlation between the BLAC determined from POCT and that from the laboratory analysis. We found a mean bias with the POCT BLAC, which was on average $0.228 \mathrm{mmol} / \mathrm{L}$ lower than that determined by laboratory analysis. This bias is consistent with a previous study comparing lactic acid levels determined using the i-STAT and laboratory analysis, respectively, in non-cancer patients in an ED setting (mean difference of 0.32 , with reasonable limit agreements) [8].

In terms of the categorization of BLAC when the blood for both tests was drawn within $2 \mathrm{~h}$ of each other, in 178 patients $(64.96 \%)$ the BLAC range from both POCT and laboratory analysis directly matched in terms of low, normal, or high. Sixty-four $(23.36 \%)$ patients were identified with normal BLAC according to the laboratory analysis and low BLAC by POCT. This result is in line with the bias on the BLAC determined by POCT being on average $0.228 \mathrm{mmol} / \mathrm{L}$ lower that that determined by laboratory analysis. Possible explanations for this bias are the assay techniques used when running the tests and the timing of the tests. POCT was performed before the laboratory analysis for most patients, and this later processing of the laboratory analysis could have inflated the resulting BLAC and thus created the bias. Twenty-eight $(10.22 \%)$ patients were identified with normal BLAC according to the laboratory analysis and high BLAC by POCT; in 21 of these patients the difference in test value between the laboratory analsis and POCT was $\leq$ $0.2 \mathrm{mmol} / \mathrm{L}$, and a chart review of these patients did not reveal any clinical sign of sepsis or any 
other condition associated with an elevated BLAC. Of the remaining seven patients in this category, a chart review showed that three patients had symptoms and clinical signs of sepsis. Finally, four $(1.46 \%)$ patients were identified with high BLAC according to the laboratory analysis and normal BLAC by POCT. A chart review of these four patients showed that two of them showed symptoms and clinical signs of sepsis. This categorization shows that POCT for lactic acid levels would have accurately predicted the risk of sepsis in 272 of the 274 patients in our study population.

The correlation and categorization of BLAC from both testing methods show that POCT can accurately measure blood lactic acid values in cancer patients. This finding is consistent with those from previous studies that have shown that a POC device can provide reliable blood lactic acid measurements in ED and delivery rooms [8-10]. The assessment of lactic acid by POCT in cancer patients can timely identify who is at risk for sepsis. Singer et al. have shown that bedside lactate POC testing in the ED in patients with suspected sepsis is associated with reduced time to treatment with intravenous fluids and decreased mortality [12].

\section{Limitations}

We acknowledge a number of limitations to this study. First, convenience sampling may have biased our results as patients were included only if they had had blood drawn for both POCT and laboratory analysis within at most $2 \mathrm{~h}$ of each other. Second, we used the time between the drawings of blood samples for POCT and laboratory analysis as reference so any significant delay between the drawing of the blood samples and actual tests could influence our analysis. Third, since the blood samples for both testing methods were drawn within $2 \mathrm{~h}$ of each other, but not necessarily drawn at the same time for some of the patients, any treatment that the patient may have received could have impacted the outcome. Fourth, we use linear regression to compare lactic acid levels determined by POCT and laboratory analysis in our paired samples.

\section{CONCLUSION}

The results of this study shown that POCT can provide accurate lactic acid values in patients with cancer and with this knowledge that the assessment of BLAC by POCT is reliable, we expect to see reduced time to triage and appropriately treat cancer patients with suspected sepsis. Furthermore, as already mentioned, POC devices are not limited to the measurement of an analyte such as lactic acid. With the integration of biosensors, they can also measure nucleic acids, proteins, circulation cells, and cytokines such as interleukin-3 (IL-3). IL-3 has been shown to be an early diagnostic marker in sepsis [13] and can therefore be used in cancer patients when there is a high suspicion of sepsis and normal lactic blood levels. Nucleic acids, proteins, and circulation cells are also vital laboratory values when evaluating cancer progression or tumor burden of patients presenting to the ED.

While patient wait time is the ED was not a focus of this study, our findings complement those of other studies showing that POCT reduces care time and length of stay of stable adult patients in the ED $[14,15]$. The decreased time in the ED for cancer patients that would come from using values obtained solely from POCT is of critical importance as those patients often need continuous treatment while in the ED. A faster discharge from the ED would decrease the patient's exposure to infection.

\section{ACKNOWLEDGEMENTS}

We thank the anonymous patients who contributed to this study and entrusted us with their care.

Funding. No funding or sponsorship was received for this study or publication of this article.

Editorial Assistance. Editorial support was provided by Bryan Tutt in Scientific 
Publications Services, Research Medical Library, UT MD Anderson Cancer Center.

Authorship. All named authors meet the International Committee of Medical Journal Editors (ICMJE) criteria for authorship for this article, take responsibility for the integrity of the work as a whole, and have given their approval for this version to be published.

Disclosures. Dr. Lavinia Middleton is on the editorial board of Oncology and Therapy but has nothing further to disclose. The remaining authors, Mr. Ron Phipp, Dr. Carmen Gonzalez, and Mr. Georges Bouobda Tsemo, have nothing to disclose.

Compliance with Ethics Guidelines. This study is a laboratory-based quality improvement study based on anonymous patient data, and as such, not on research on human subjects, and is therefore exempt from review by our institution IRB.

Data Availability. The datasets during and or analyzed during this current study are available from the corresponding author on a reasonable request.

Open Access. This article is licensed under a Creative Commons Attribution-NonCommercial 4.0 International License, which permits any non-commercial use, sharing, adaptation, distribution and reproduction in any medium or format, as long as you give appropriate credit to the original author(s) and the source, provide a link to the Creative Commons licence, and indicate if changes were made. The images or other third party material in this article are included in the article's Creative Commons licence, unless indicated otherwise in a credit line to the material. If material is not included in the article's Creative Commons licence and your intended use is not permitted by statutory regulation or exceeds the permitted use, you will need to obtain permission directly from the copyright holder. To view a copy of this licence, visit http://creativecommons.org/licenses/bync/4.0/.

\section{REFERENCES}

1. Trzeciak S, et al. Serum lactate as a predictor of mortality in patients with infection. Intensive Care Med. 2007;33(6):970-7.

2. Hsu PP, Sabatini DM. Cancer cell metabolism: warburg and beyond. Cell. 2008;134(5):703-7.

3. Bakker J, Nijsten MWN, Jansen TC. Clinical use of lactate monitoring in critically ill patients. Ann Intensive Care. 2013;3:12-12.

4. Dai Y, Liu CC. Recent advances on electrochemical biosensing strategies toward universal point-of-care systems. Angew Chem Int Ed. 2019;58(36): 12355-68.

5. Dai Y, et al. Exploring the trans-cleavage activity of CRISPR-Cas12a (cpf1) for the development of a universal electrochemical biosensor. Angew Chem Int Ed. 2019;58(48):17399-405.

6. Sage AT, et al. Ultrasensitive electrochemical biomolecular detection using nanostructured microelectrodes. Acc Chem Res. 2014;47(8): 2417-25.

7. Poudineh $\mathrm{M}$, et al. Profiling functional and biochemical phenotypes of circulating tumor cells using a two-dimensional sorting device. Angew Chem Int Ed. 2017;56(1):163-8.

8. Shapiro NI, et al. The feasibility and accuracy of point-of-care lactate measurement in emergency department patients with suspected infection. J Emerg Med. 2010;39(1):89-94.

9. Orsonneau J-L, et al. Suitability of POC lactate methods for fetal and perinatal lactate testing: considerations for accuracy, specificity and decision making criteria. Clin Chem Lab Med. 2013;51:397.

10. van Horssen R, et al. Lactate point-of-care testing for acidosis: cross-comparison of two devices with routine laboratory results. Pract Lab Med. 2016;4: $41-9$.

11. Gaieski DF, et al. Impact of time to antibiotics on survival in patients with severe sepsis or septic shock in whom early goal-directed therapy was initiated in the emergency department. Crit Care Med. 2010;38(4):1045-54.

12. Singer AJ, et al. ED bedside point-of-care lactate in patients with suspected sepsis is associated with reduced time to iv fluids and mortality. Am J Emerg Med. 2014;32(9):1120-4. 
13. Min J, et al. Integrated biosensor for rapid and point-of-care sepsis diagnosis. ACS Nano. 2018;12(4):3378-84.

14. Jang JY, et al. Use of a comprehensive metabolic panel point-of-care test to reduce length of stay in the emergency department: a randomized controlled trial. Ann Emerg Med. 2013;61(2):145-51.

15. Singer AJ, et al. Early point-of-care testing at triage reduces care time in stable adult emergency department patients. J Emerg Med. 2018;55(2): $172-8$. 